\title{
A new mandibular anchorage solution inspired from alpinism
}

\author{
C. Bernard-Granger ${ }^{1}$, P. Cresseaux ${ }^{2}$, R. Filippi ${ }^{1}$ \\ 1 Specialist certified in Orthodontics and Dentofacial Orthopedics, private practice, Lyon \\ 2 Maxillofacial surgeon - Lyon
}

\section{ABSTRACT}

In this article, we are presenting a new mandibular anchorage solution through three clinical cases.

This method is based on a mountain climbing technique. Thanks to a simple fixation wire insert in the jaw bone, it is now possible to obtain a backward movement of the mandibular arch and a significant uprighting of the incisors.

\section{KEYWORDS}

Anchorage, distalization, incisor uprighting, fixation wire, jaw bone

\section{INTRODUCTION}

Posterior anchorage on the mandibular arch during orthodontic treatment has always been difficult to achieve. We have tested a lot of anchorage screws and plate systems that gave us quite acceptable clinical results, but they had many inherent disadvantages.

The area surrounding the retromolar trigone, a common area for posterior anchorage, has a thick mucosa and is complicated to access. Furthermore, orthodontists often have difficulty reactivating orthodontic appliances. Such systems pose problems for patients with frequent injuries, because of whom the number of emergency appointments increases significantly in our practices. One final disadvantage is that all these systems require surgical reintervention at the time of removal because they have to be buried in order to be solidly anchored distally of the mandibular teeth. However, this area is a prime site for orthodontic anchorage because distancing it from the mandibular molars opens up new possibilities in many treatment plans.

We were inspired by a technique developed in the 1930s by a Soviet mountaineer, Vitaly Abalakov. He revolutionized mountaineering by developing an infallible anchorage system in the ice, which allowed him to climb Lenin Peak and Khan Tengri mountain (two peaks above 7,000 $\mathrm{m}$ in altitude in the Caucasus).

This technique, which bears his name, represented a veritable revolution in glacial

Address for correspondence:

Chloé Bernard-Granger - 4 allée du

Champ de Maïs - 74940 - Annecy-le-Vieux

E.mail: dr.bernardgranger@gmail.com

This is an Open Access article distributed under the terms of the Creative Commons Attribution License (http://creativecommons.org/licenses/by/4.0), which permits unrestricted use, distribution, and reproduction in any medium, provided the original work is properly cited. 


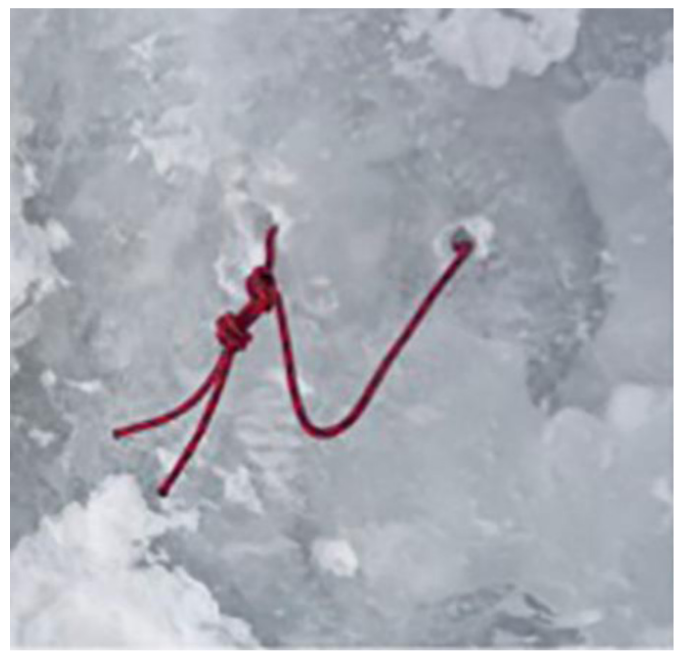

Figure 1

Final placement of the anchorage, front view. The cord is secured by an ice bridge.

mountaineering. It is an impeccable anchorage system in the ice, ensuring the life and survival of the mountaineer. This system is easy to implement, inexpensive (requires only a piece of rope), and ultimately leaves no trace in the ice.

It is a very simple anchor since a connection is easily made by passing a rope through two holes close together in a block of ice. It is extremely reliable thanks to the ice bridge which secures the rope.

We have logically tried to adapt this mountaineer's amazing idea to resolve our issues with posterior anchorages on the mandible.

In this article, we are proposing a new orthodontic anchorage which we hope, like Abalakov's in the 1930s, will certainly revolutionize dental anchorage systems. With this technique, the posterior mandibular anchorage will be stable, reliable and inexpensive. It would not require surgery, can be reactivated easily, and above all, it is very comfortable.
This article will also present some examples of molar distalization performed on patients using this technique.

\section{Clinical Case 1}

Our first patient is a 19-year-old young man who presents with a skeletal class-II malocclusion with mandibular retrusion.

The intraoral examination showed a corrected transverse alignment. In the sagittal direction, there is a class-I canine malocclusion, and the right and left molars (36 and 46 respectively) were crowned with pedodontic caps.

A radiographic exam revealed an incomplete endodontic treatment on tooth 46.

Cephalometric examination confirms the diagnosis of a skeletal class-II mandibular retrusion and a high degree of vestibuloversion of the mandibular incisors. The maxilla is well positioned and the maxillary dental axes are aligned normally.

The chosen treatment is:

- The extraction of 46; 46 cannot be retained for endodontic reasons. Its mesiodistal diameter will be maintained so that it can be replaced by an implant at the end of treatment;

- the extraction of the third mandibular molars;

- the placement of a temporary crown on 36 (in order to preserve as much dental tissue as possible and to regain a suitable mesiodistal size);

- surgical orthodontic procedure with mandibular advancement surgery. 

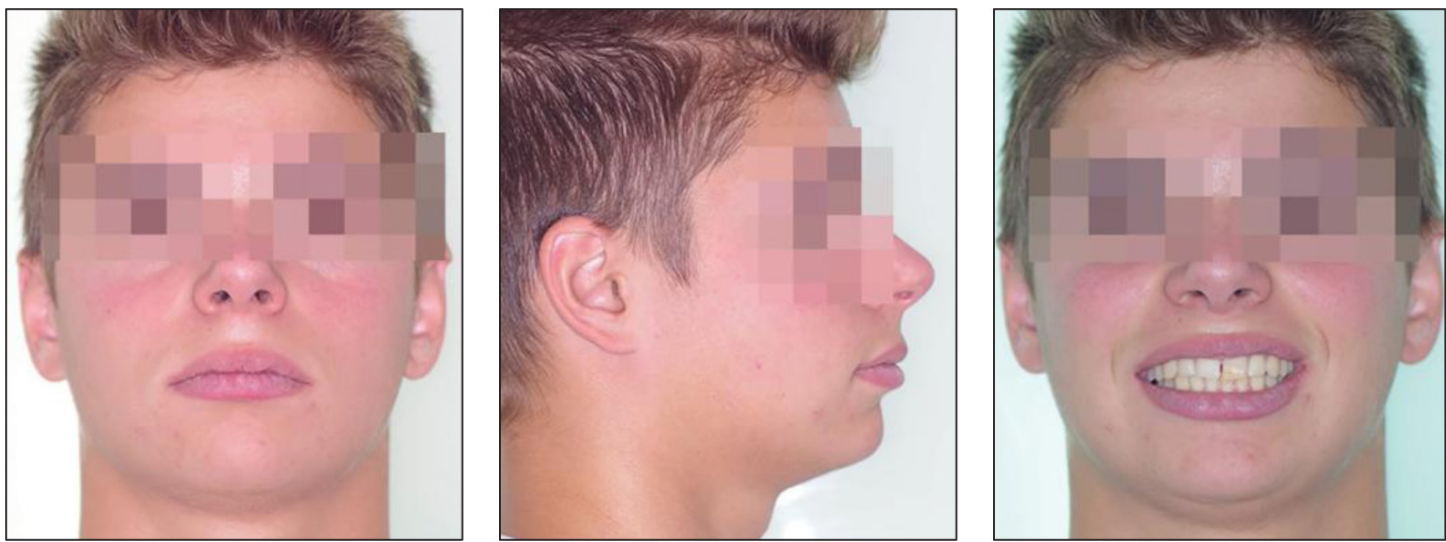

Figures $2 a, b, c$

Extraoral examination.
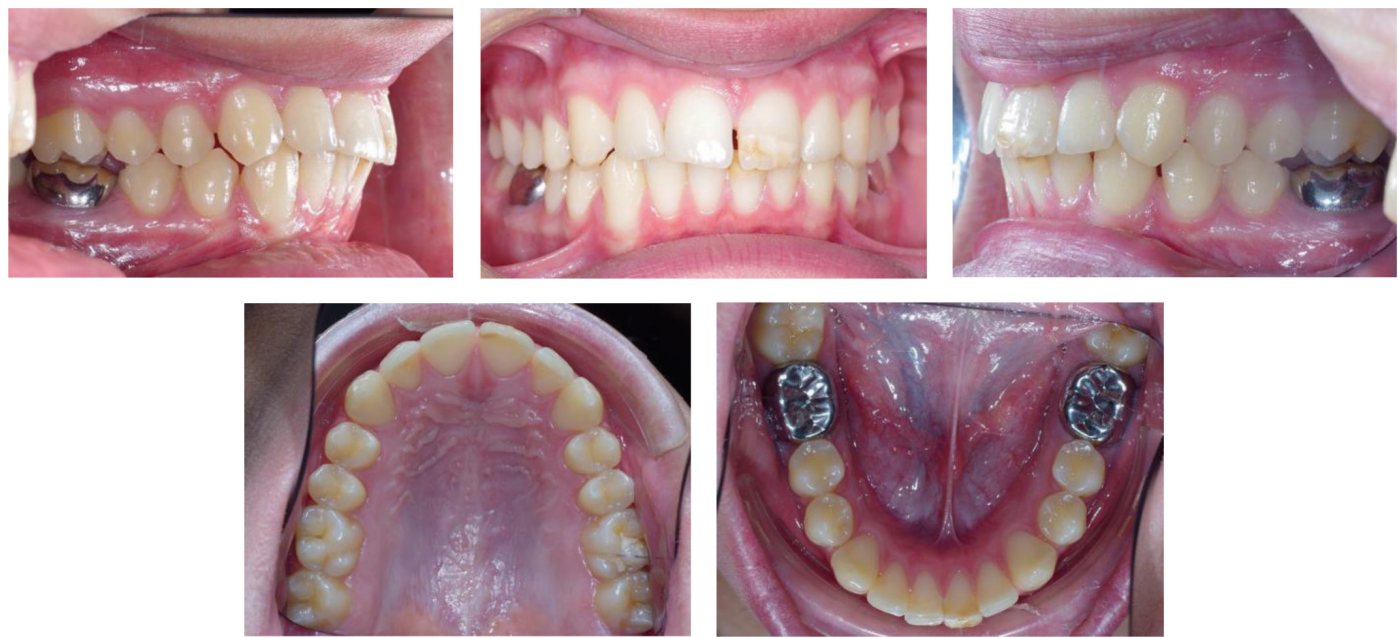

Figures $3 a, b, c, d, e$ Intraoral examination.

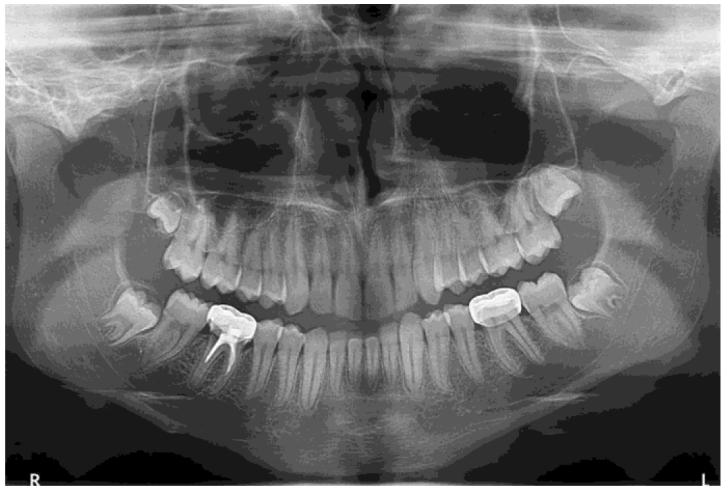

Figure 4

Orthopantomogram at the start of treatment.

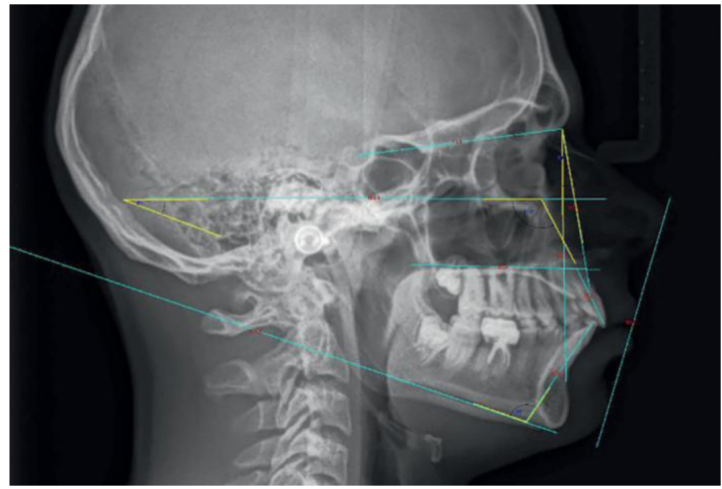

Figure 5

Cephalometric exam at the start of treatment. 
In this situation the role of the orthodontist is to decompensate only the mandibular arch to prepare the patient for surgery.

Preoperative decompensation aims to restore correct mandibular incisal axes and a class-II canine and molar malocclusions by retracting the mandibular arch exclusively without any damage to the maxillary arch.

Therefore, we decided to adapt Vitaly Abalakov's technique to create a new posterior mandibular anchorage.

This simple solution involves the use of a standard wire fixation procedure and will allow us to decompensate lower arch alone.

The patient is fitted with an appliance that is a maxillary and mandibular vestibular multibracket system.

To achieve posterior mandibular anchorage, the wire fixation is positioned during the extraction of the all third molars.

\section{THE SURGICAL PROCEDURE}

These are basic fixation wires that were used 20 years ago before the advent of plate fixation.

They are placed at half the coronal height of the molars, which corresponds to the bonding height of the multibracket appliance.

These wires can be inserted under local anesthesia (an intravenous sedation can be used for supplementation). Access to the mandibular ramus is equivalent to the access gained by extracting the four third molars. These are often extracted during such operations as they improve the retraction of the lower arch and consequently the incisal adjustment.

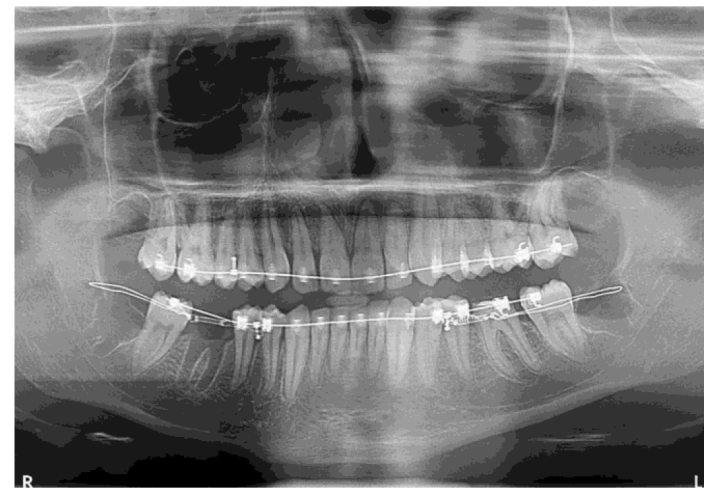

Figure 6

Orthopantomogram taken when applying traction of the system.

A perforation is created in the ascending ramus, under irrigation, using a round bur with handpiece attachment, to allow the wire fixation to pass on both sides, thereby avoiding lingual nerve injury.

A fixation wire, $0.3 \mathrm{~mm}$ in diameter, is then inserted in the perforation. Both strands will be twisted and folded on

the orthodontic appliance during the surgical procedure so as not to cause any discomfort to the patient. The orthodontist can apply traction to the system immediately after its installation.

Postoperative management is straightforward and most often is similar to standard third molar extractions.

The anchorage is put in traction with the use of a simple 200-g NiTi spring that is easily placed after the surgery. The posterior end of the spring is attached to the twisted portion of the fixation wire. The front end of the spring is connected to the lower multiband appliance by way of metallic suture ties. Thus, this creates direct traction. 
We start distalization in our patients using of a $0.020 \times 0.020$ shape memory alloy.

An elastometric chain is placed on the anterior teeth to obtain a complete retraction of the entire arch.
On the right side, a Torque Albaflex coated archwire is placed to maintain the space designated for implant placement in position 46 at the end of treatment.
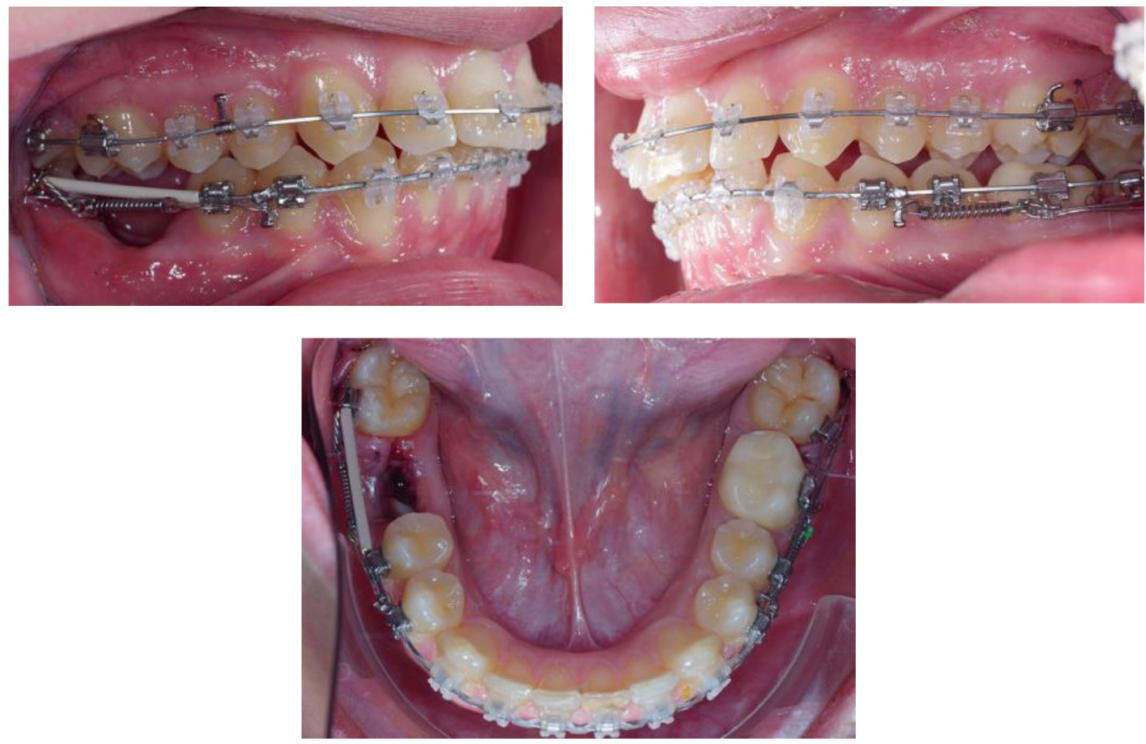

Figures $7 a, b, c$

Right, left, and occlusal views of the traction system.
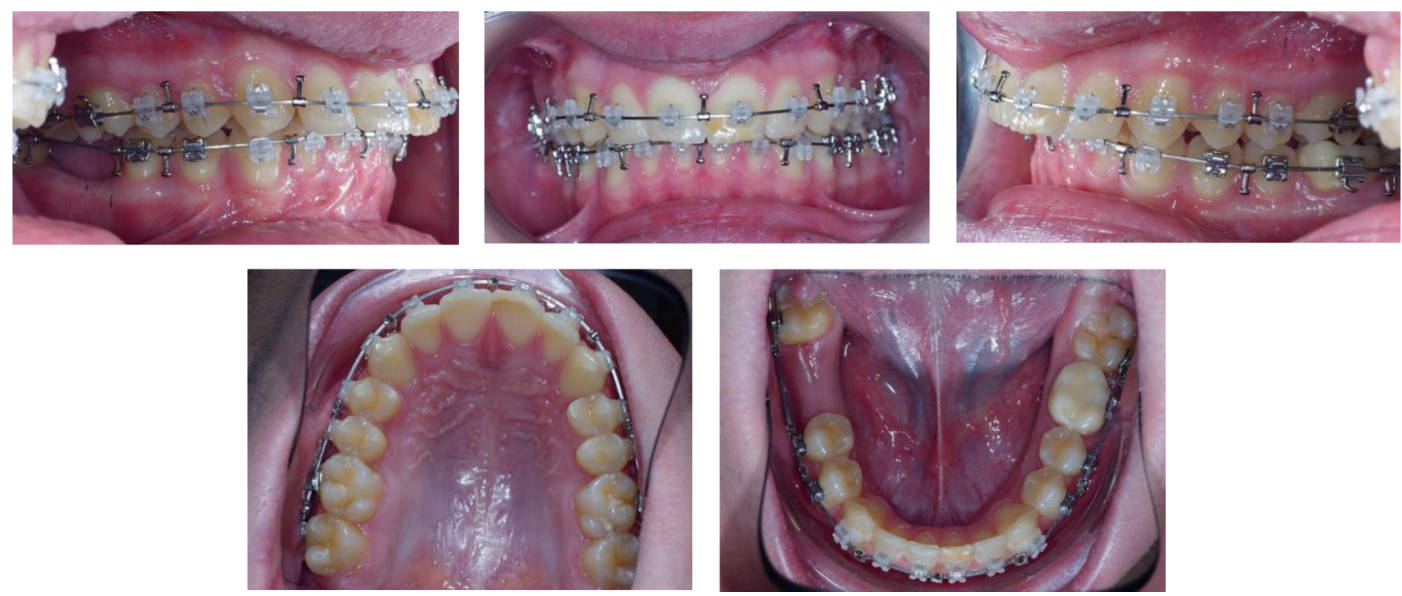

Figures $8 a, b, c, d, e$

Intraoral photographs at the end of the orthodontic preparation phase. This is 5 months after the start of treatment. 


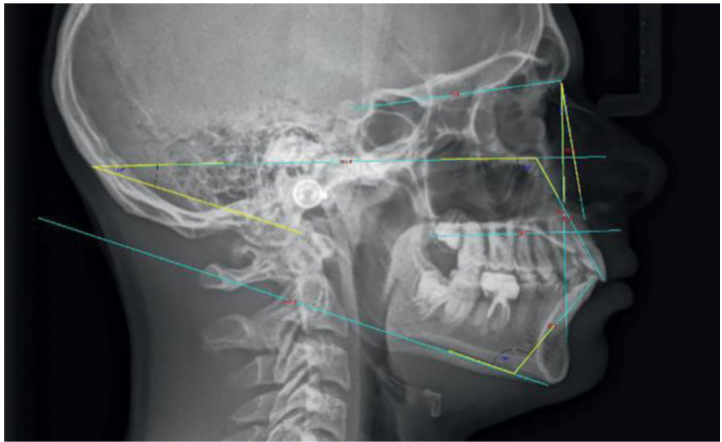

Figure 9

Profile teleradiography at the start of treatment.

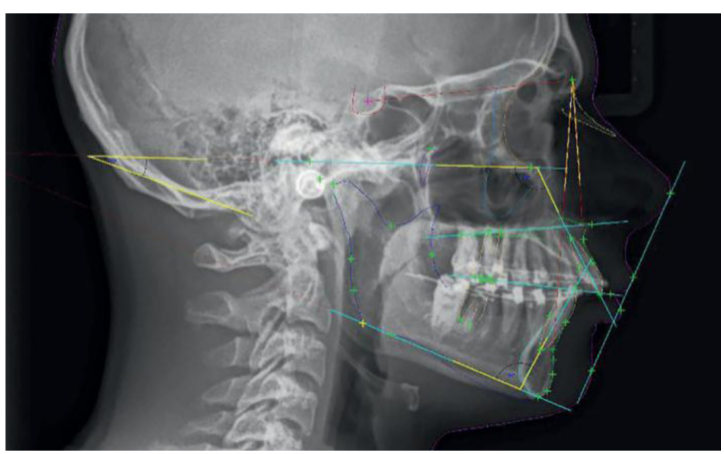

Figure 10

Profile teleradiography in May 2016 lafter 5 months of traction on the wire anchorage in the mandibular ascending ramus).

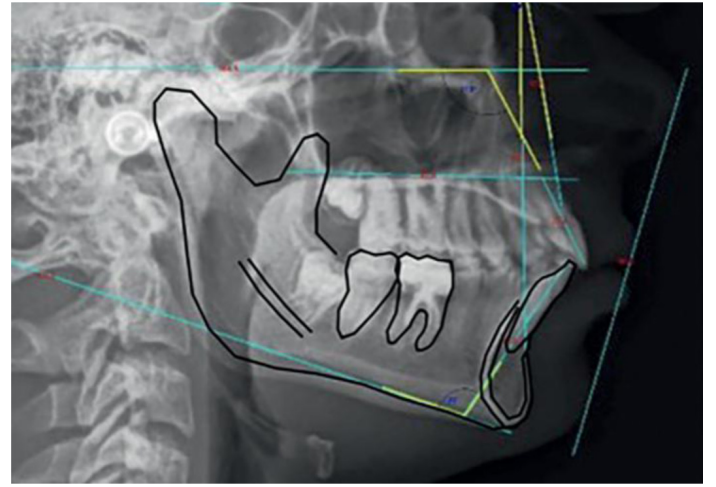

Figure 11

Cephalometric examination from the start of treatment.

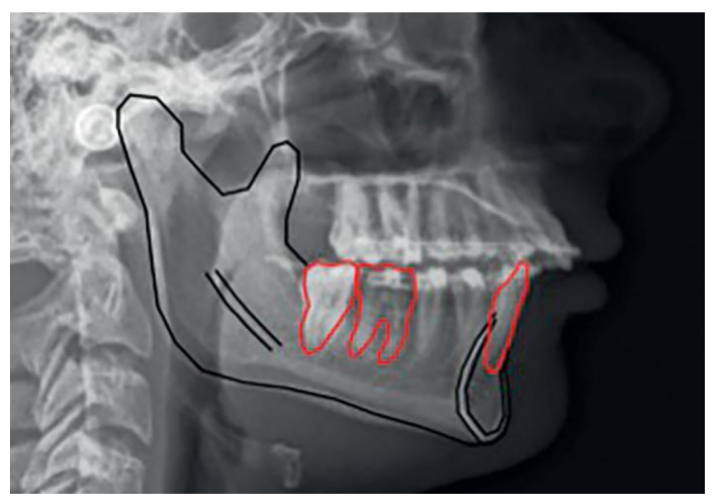

Figure 12

Cephalometric examination at the end of the orthodontic preparation phase.

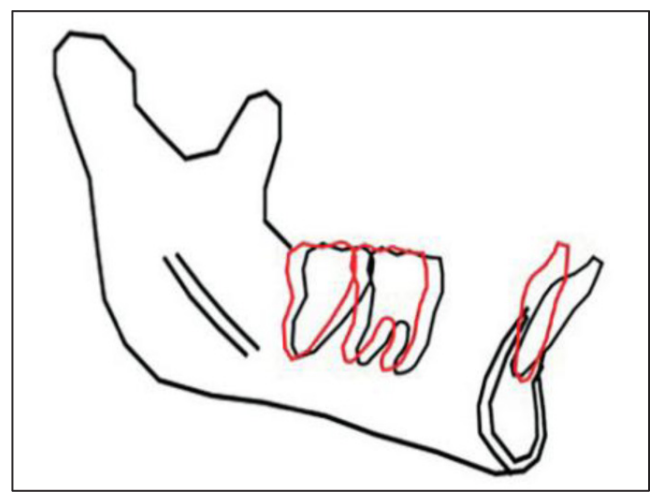

Figure 13

Local mandibular superimposition between the start of treatment and the end of orthodontic preparation (5 months between images). An adjustment of the incisal axis and molar distalization are observed. 
The first results were obtained quickly. The traction is then reactivated 1 month after the first setting in traction by simply moving the front part of the spring and a TMA archwire measuring $0.019 \times 0.025$, is inserted.

After 5 months of traction, the desired incisal adjustment was obtained, and we schedule the mandibular advancement surgery.

A comparison of the $x$-rays makes it possible to objectify the mandibular incisal adjustment and the mandibular molar distalization obtained. We also observe the maintenance of the incisal axes at the level of the maxillary arch.

A very significant incisal adjustment is observed. Indeed, the incisal axis increased from $112^{\circ}$ to $92^{\circ}$ in 5 months. A molar distalization of $3 \mathrm{~mm}$ was also obtained. The maxillary incisal axes have been maintained, which aligns perfectly with our initial objective.

On the orthopantomogram, the remnant alveolus after the extraction of 46 (at the beginning of orthodontic treatment) proves to be an excellent radiographic marker. The dental alveolus

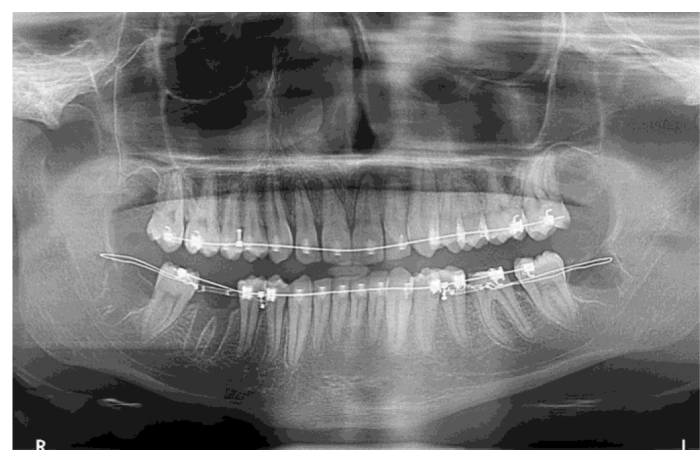

Figure 14

Orthopantomographic examination during placement of the mandibular wire anchorage.

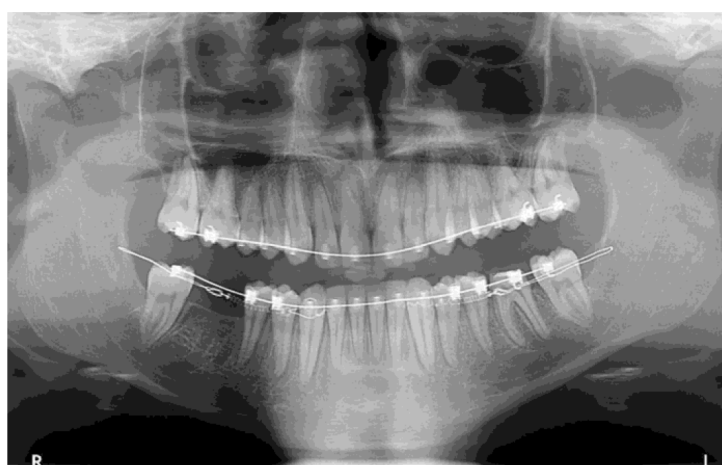

Figure 15

Orthopantomographic examination at the end of the surgical preparation phase.
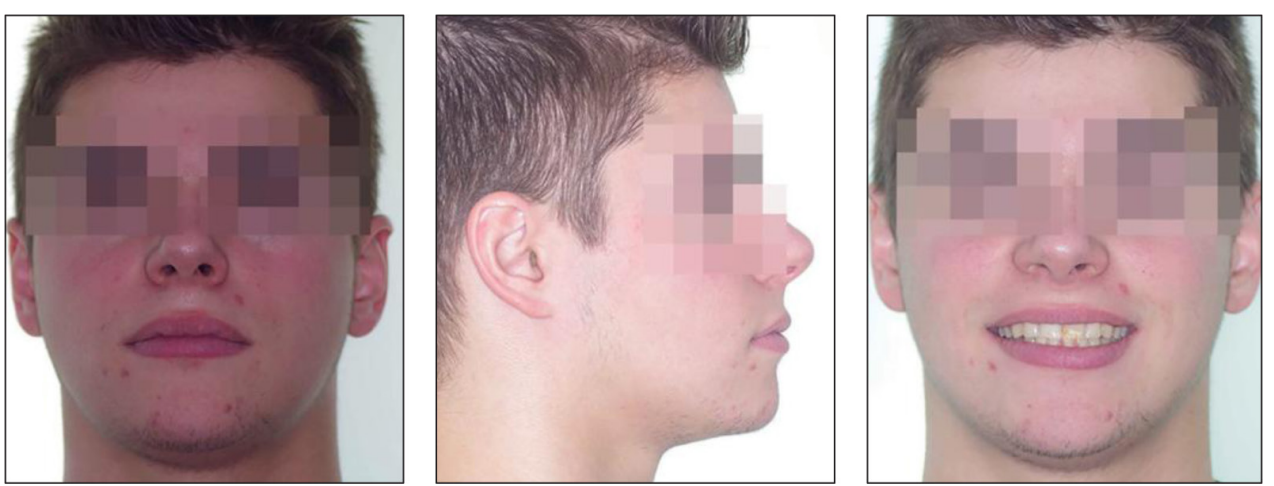

Figures 16a, b, c

Extraoral photographs after treatment. 

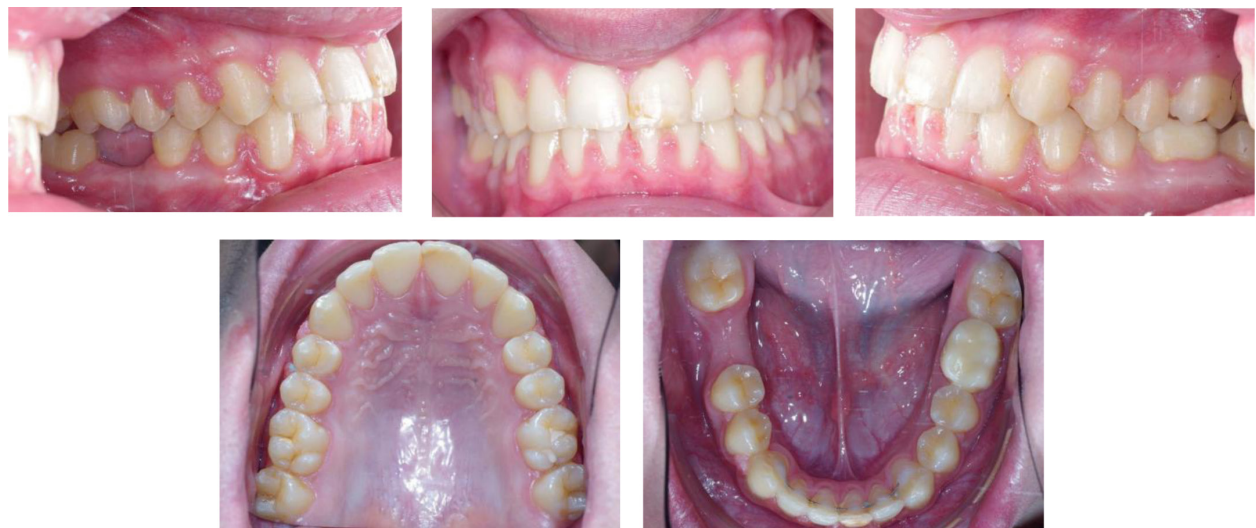

Figures $17 a, b, c, d, e$

Intraoral photographs at the end of treatment.

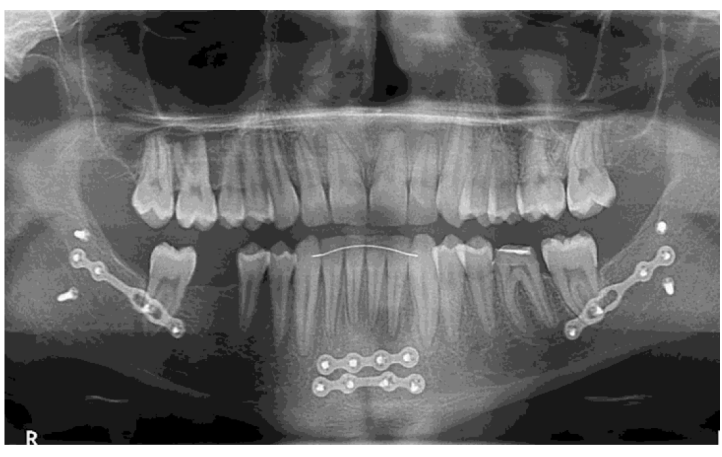

Figure 18

Orthopantomographic examination at the end of treatment.

behaves like a Björk marker (used in his studies on growth directions). Since, the alveolus is not displaced by the orthodontic treatment, it constitutes a stable radiological reference point. On the orthopantomogram at the initiation of traction, we note the retraction of 47 and 45 in relation to the phantom space represented by the former alveolus of 46.

Throughout the treatment, the patient did not complain of any discomfort caused by the traction system and he even described the experience as "comfortable." The system was installed during orthognathic surgery.

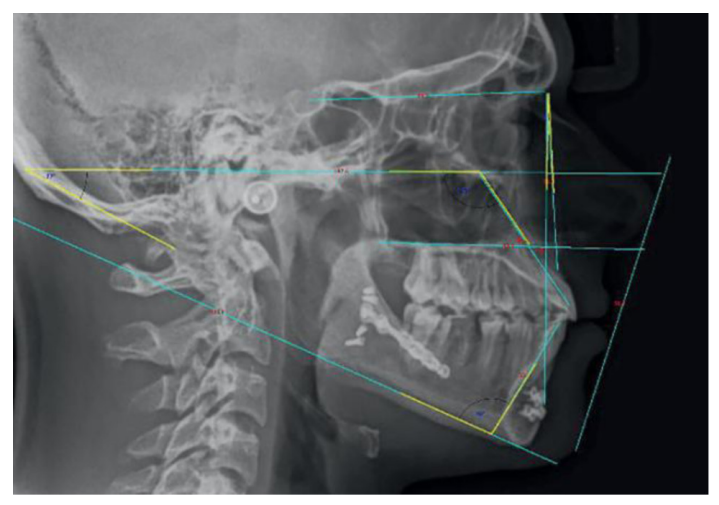

Figure 19

Cephalometric examination at the end of treatment.

The operation was performed by Dr. Paul Cresseaux, in the seventh month of treatment. It consisted of a simple mandibular advance.

Owing to the favorable adjustment of the incisal axes and a subsequently effective preoperative decompensation phase, sufficient mandibular advancement was achieved, and the skeletal and dental class-II malocclusions were completely corrected.

The preoperative decompensation phase occurred very quickly because of the type of anchorage provided by the mandibular wire. 
The appliance was removed in December 2016.

The total duration of this surgical orthodontic treatment was therefore 13 months.

The skeletal, dental, and functional objectives were achieved.

\section{Clinical Case 2}

This patient presents with an incisal supraclusion a DDM as well as a classII dental malocclusion on a class-II skeletal pattern. Upon clinical examination, a retrogression and significant dental compensations were also noted. The
IMPA (incisor mandibular plane angle) is $117^{\circ}$.

We chose to use Abalakov-type anchorages to decompensate the incisal axes and correct the crowding issue without extracting from the lower arch. We estimated a total duration of 18 months; the surgical stage will take place 9 months after orthodontic preparation.

The patient chose a mixed technique device with upper arch lingual braces (FKS system) and ceramic braces on the lower arch. Abalakov-type anchorages are installed a few days after the placement of our orthodontic braces.
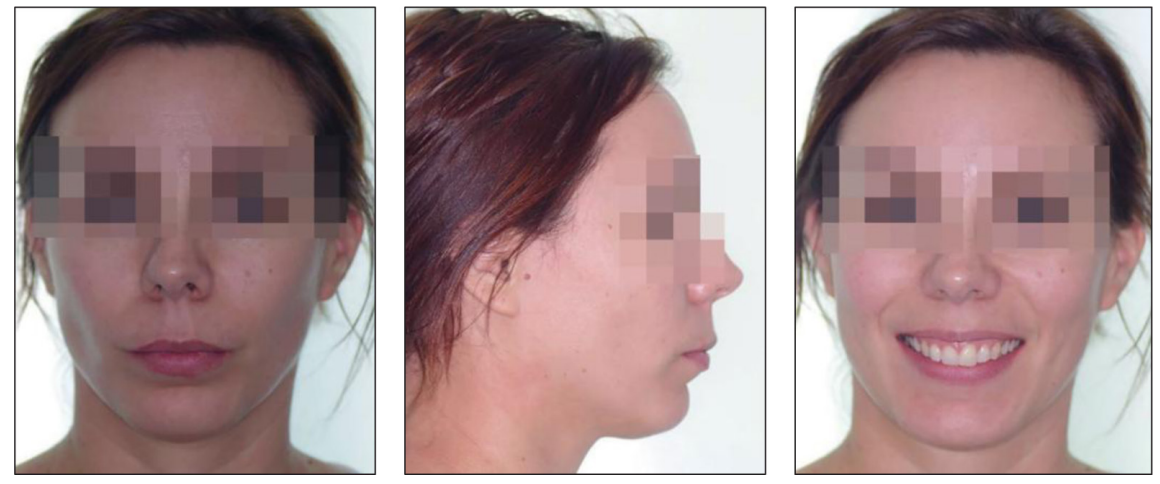

Figures 20a, b, c Extraoral examination.
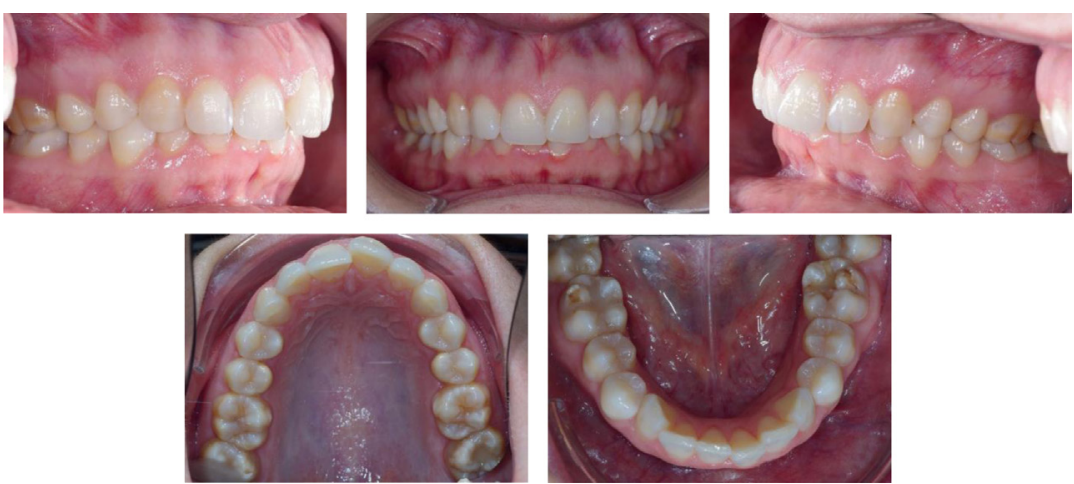

Figures 21a, b, c, d, e Intraoral examination. 


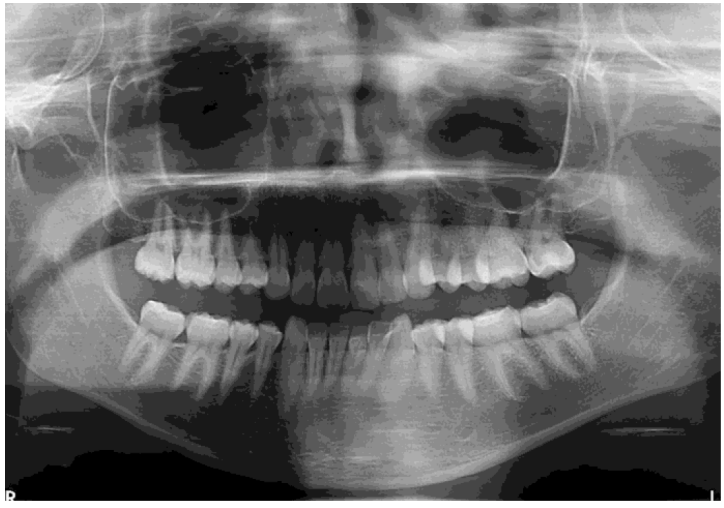

Figure 22

Orthopantomogram of the start of treatment.

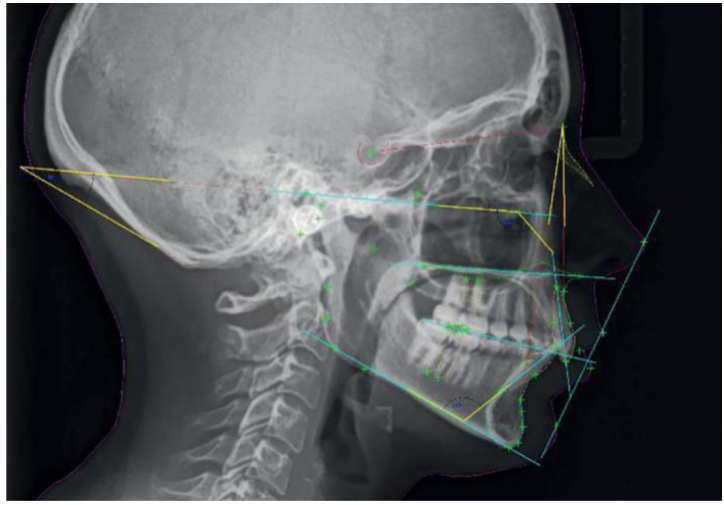

Figure 23

Cephalometric examination IMPA at $117^{\circ}$.

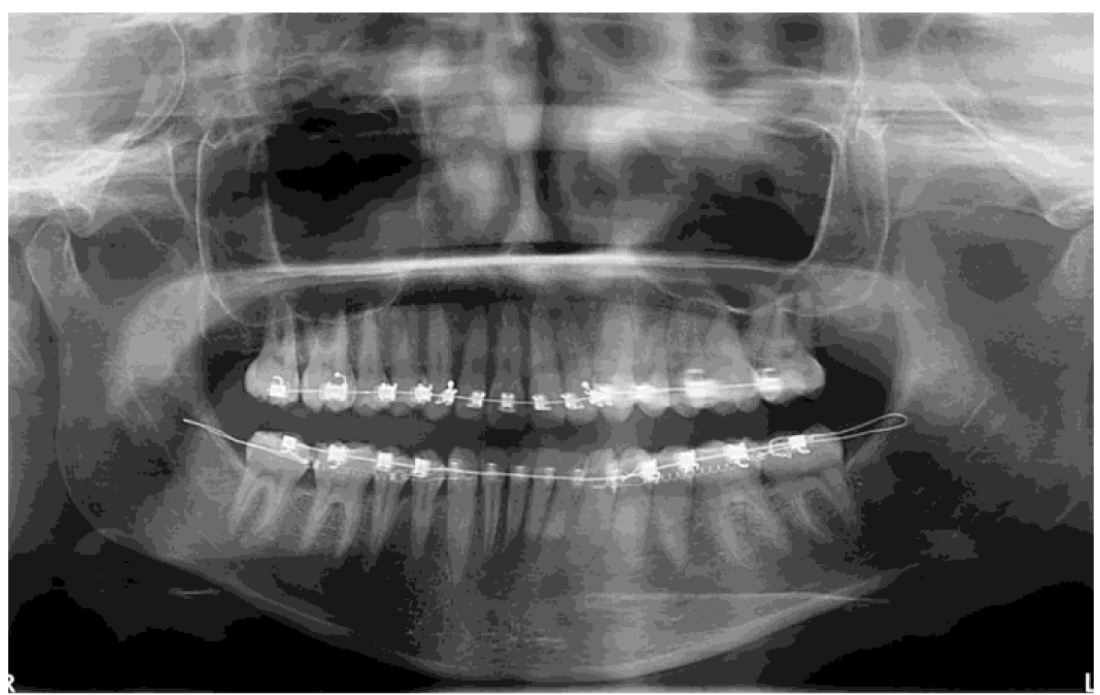

Figure 24

Setting up the Abalakov system.

The traction system was installed on the same day.

The system used is a 200-g NiTi spring that is in direct traction between the surgeon's wire and mandibular canines. It is easy to install, manipulate, and reactivate. It is also comfortable for the patient who has not complained of any pain. The postoperative consequences were nonexistent; there were no wisdom teeth.
After 6 months of traction, the incisal axis completely normalized and the crowding on the lower arch was corrected. Decompensation is optimal and the surgeon was able to peacefully contemplate his mandibular advancement procedure. The IMPA went from $117^{\circ}$ to $95^{\circ}$ within 6 months of the orthodontic preparation. 

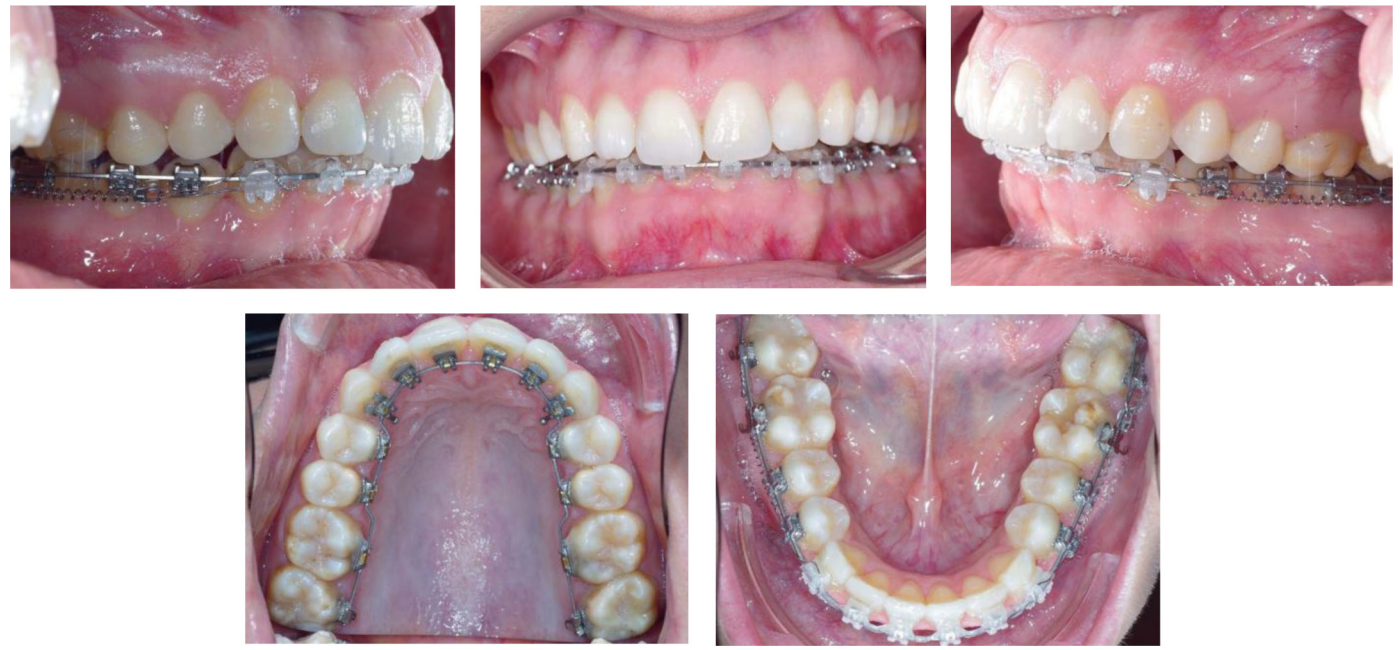

Figures 25a, b, c, d, e

Intraoral examination after the placement of the Abalakov system.

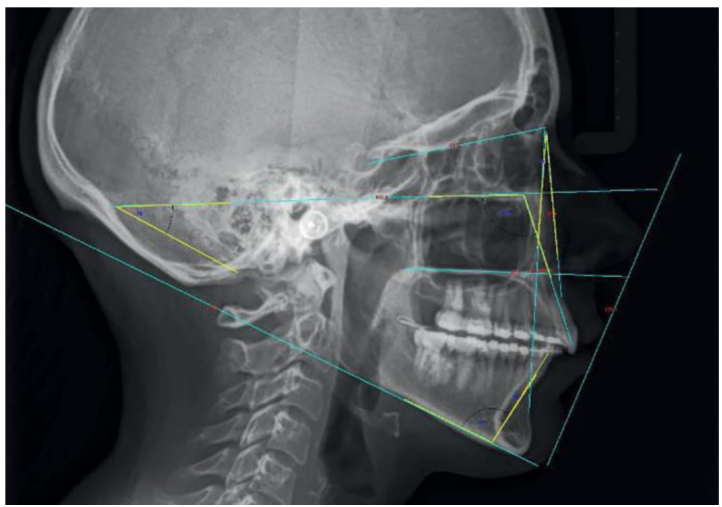

Figure 26

Profile teleradiography taken at the end of traction, preoperative reassessment, IMPA at $95^{\circ}$.

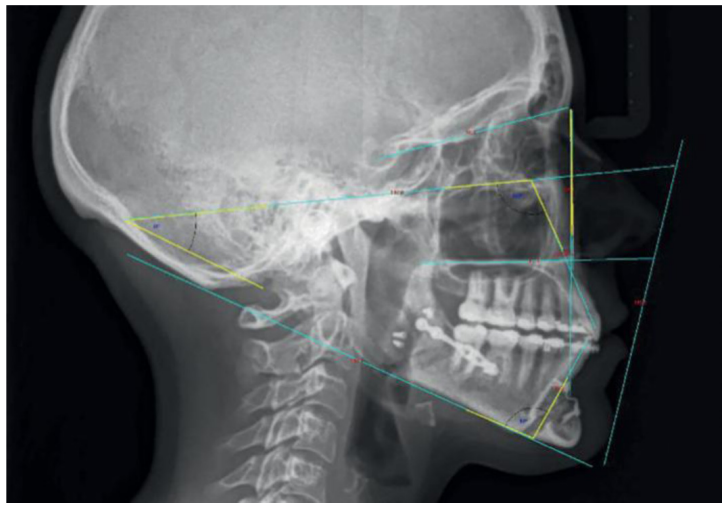

Figure 27

Post-surgical profile teleradiography.
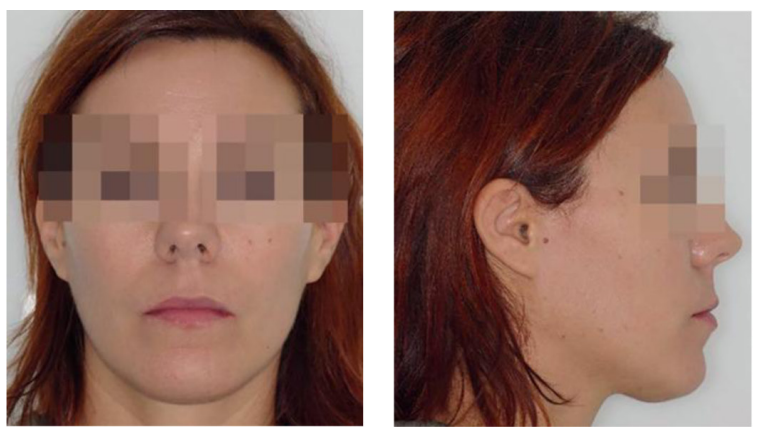

Figures 28a, b, c

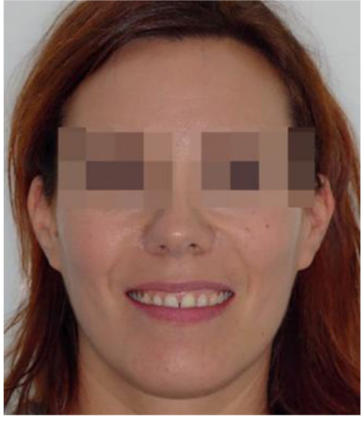

Extraoral examination at one month post-operation. 

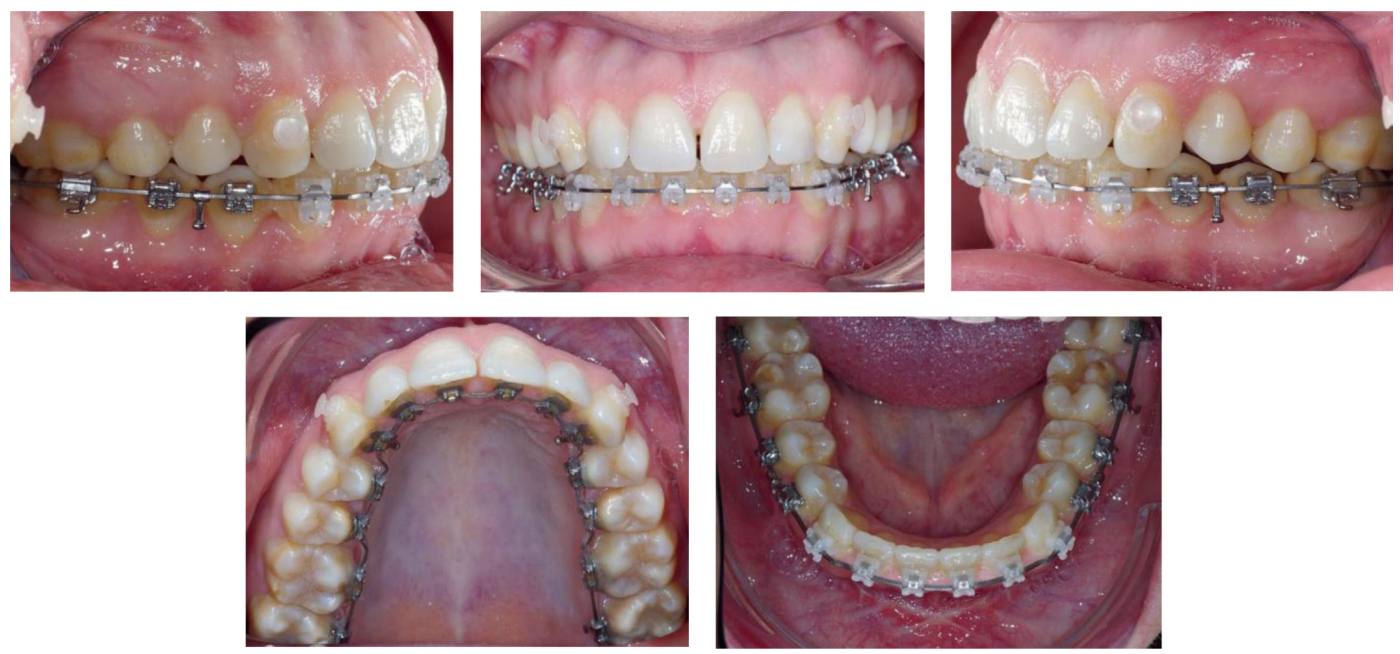

Figures 29a, b, c, d, e

Intraoral examination at 1 month post-operation.
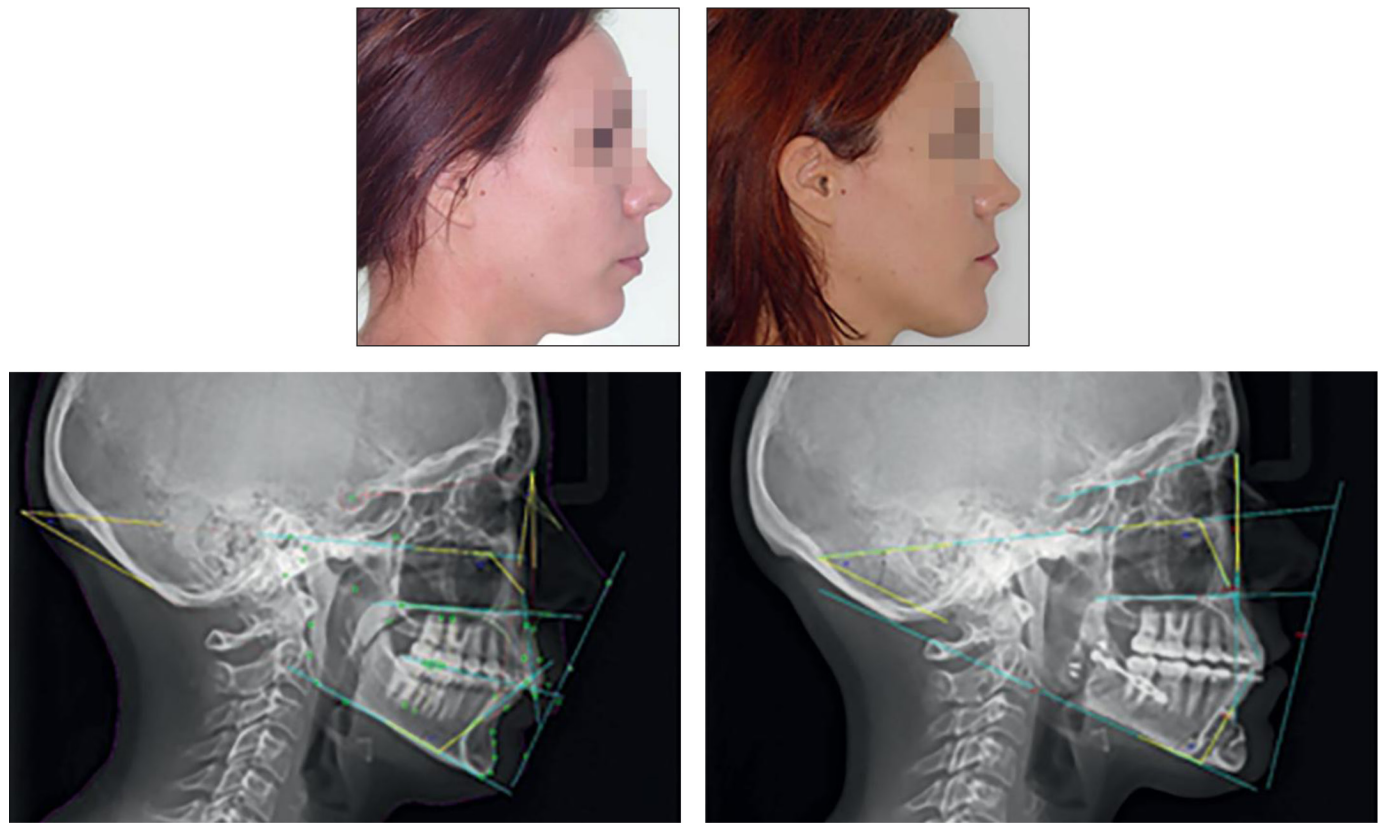

Figures $30 a, b$ and $31 a, b$

Profile photographs and profile teleradiography used to compare the start of treatment and the clinical situation 1 month after the operation. 

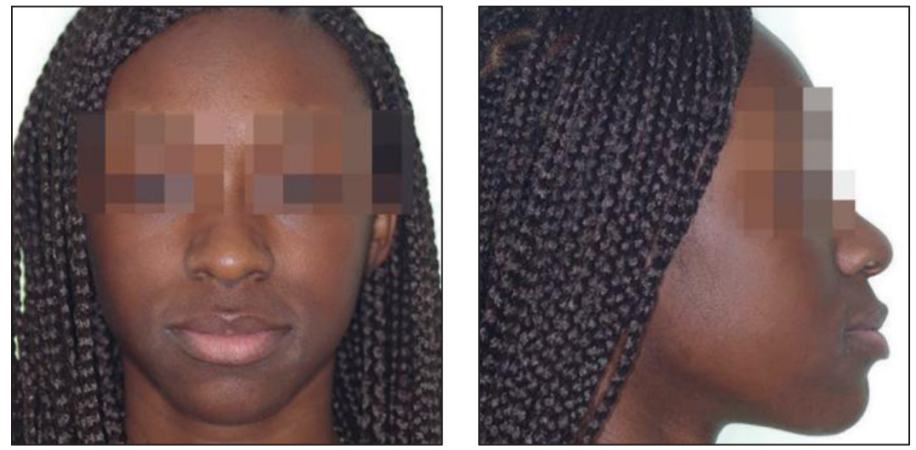

Figures $32 a, b, c$

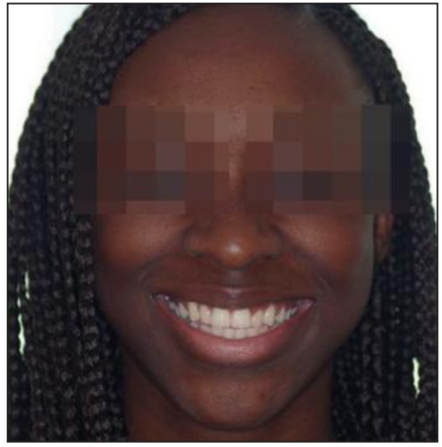

Extraoral examination at the start of treatment.
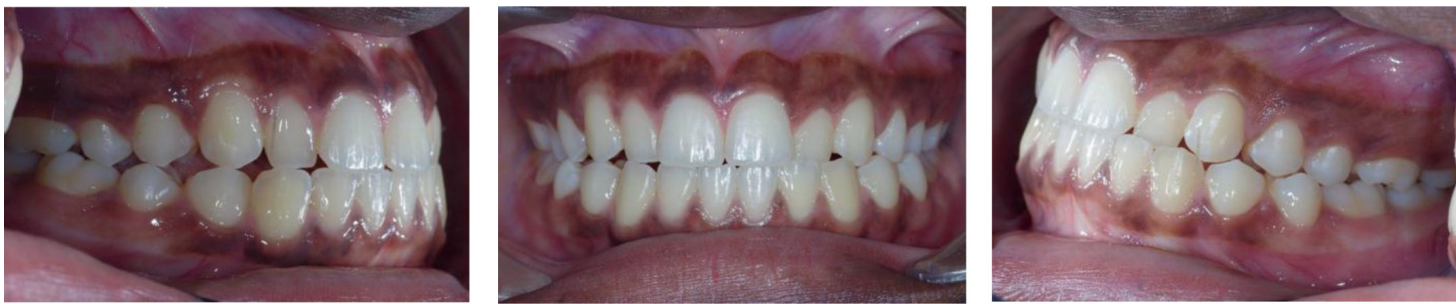

Figures 33a, b, c

Intraoral examination at the start of treatment.

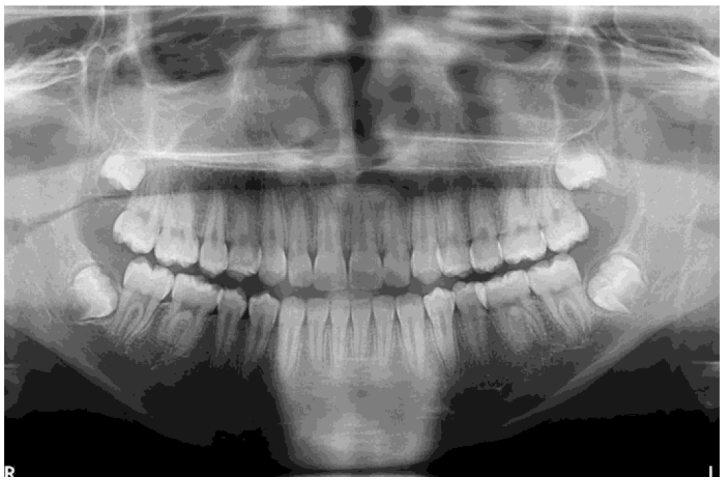

Figure 34

Orthopantomogram at the start of treatment.

\section{Clinical Case 3}

This patient came for a second opinion and she met with a practitioner who told her about maxillofacial surgery.

She presented with a dental classIII malocclusion, but after carefully

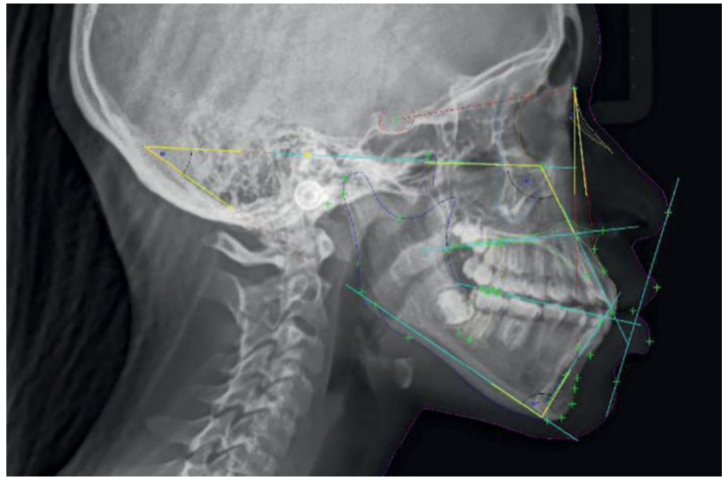

Figure 35

Cephalometry done at the beginning of treatment.

analyzing the cephalometric examination, we noticed a skeletal class-II bialveolar protrusion.

Because the patient's ethnicity, surgery was not an option. Moreover, considering the muscular and functional environment of the malocclusion, we 

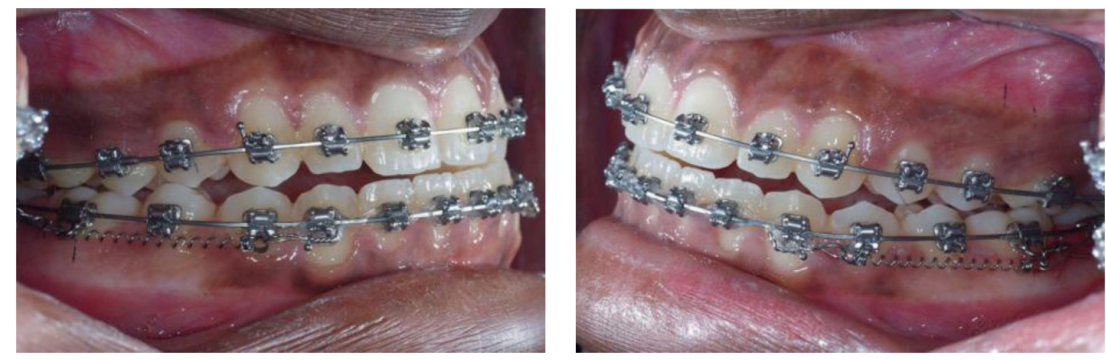

Figures 36a, b

Mandibular distalization system in place.

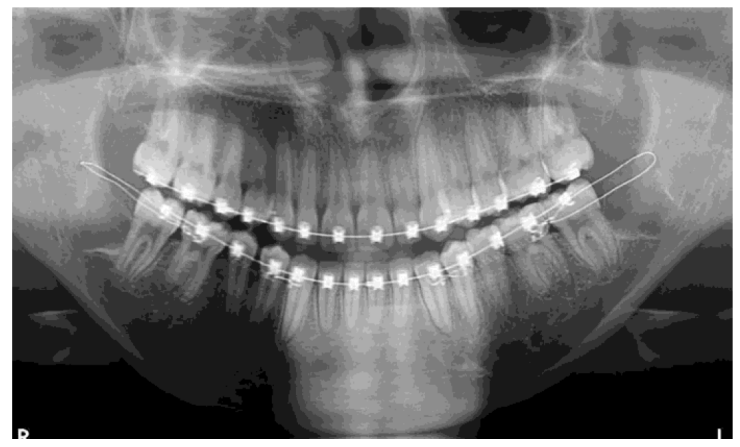

Figure 37

Orthopantomogram following the extractions of four third molars and installation of the Abalakov anchorage.
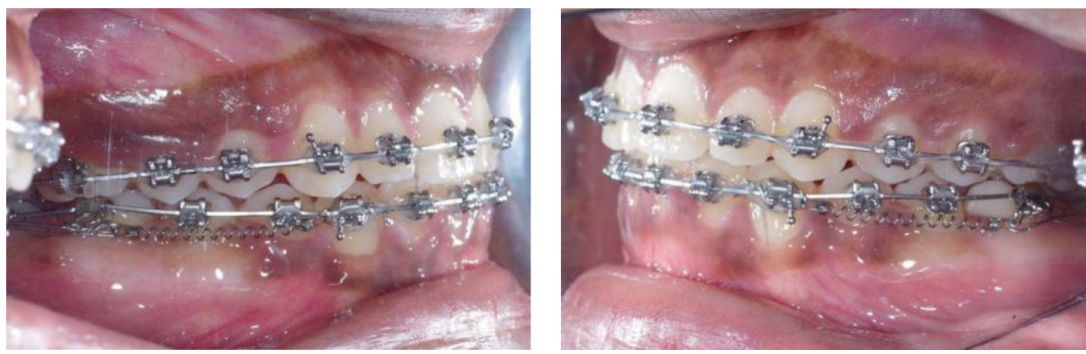

Figure 38

Evolution of the mandibular distalization after 4 months of traction.

did not propose orthodontic treatment with premolar extractions.

The four germs of the patient's third molars were still in the process of developing.

In our estimation, this patient only presented with dental dysmorphia as her profile appeared to be symmetrical to us; moreover, she did not request any aesthetic changes.

We decided to treat her dental problem as purely orthodontic and to retract the lower arch using Abalakov's method.

Dr. Paul Cresseaux extracted the four germs of the patient's third molars and 

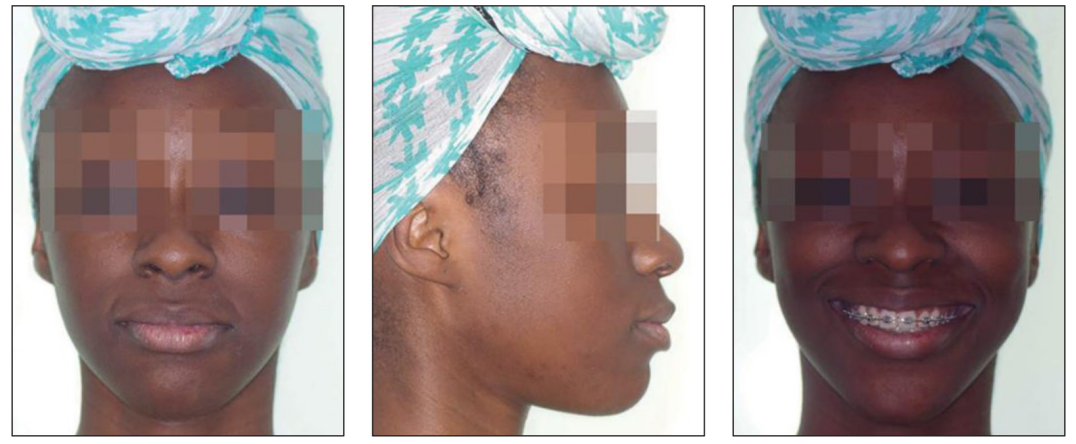

Figures 39a, b, c

Extraoral examination after 9 months of treatment.
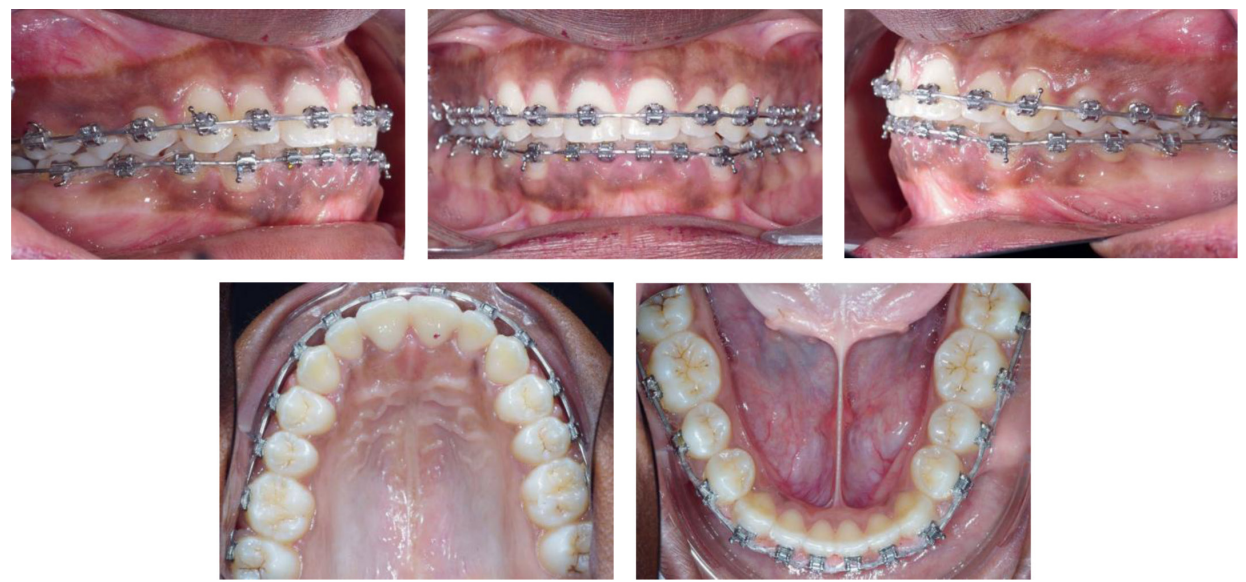

Figures $40 a, b, c, d, e$

Intraoral examination after 9 months of treatment.

placed two anchorages in the same operative phase and under local anesthesia. Traction was applied to the anchorage systems quickly and following the standard procedure of using 200-g NiTi springs in direct traction on the mandibular canines.

The entire mandibular arch was moved distally without the patient being required to wear any class-III intermaxillary elastics.

After only 9 months of treatment, the occlusion was normalized and a dental class-I malocclusion was observed. The patient also gained an ideal incisal overlap and we then asked Dr. Cresseaux to remove the anchorages.

The removal of both Abalakov-type anchorages was done with a simple local anesthesia. The wire was cut then pulled through the gum without causing any pain to the patient.

This new anchorage system perfectly meets the ideal specifications. It is reliable, easy to place, fast, patients experience no discomfort, it does not require surgical reintervention to be removed, and is inexpensive. It can be used for direct and indirect anchorage. 
Fixation wires made of surgical steel are materials that have been in used by maxillofacial surgeons for many years, and clinical perspective based on their biocompatibility has been significant.

The diameter used of $0.3 \mathrm{~mm}$ is rigid enough to achieve a massive retraction of the arch without risking breakage,

\section{CONCLUSION}

Mandibular wire anchorage brings us into a new dimension of orthodontic anchorages. As is often the case, good ideas can be found in different fields.

Thanks to this system, orthodontic preparations for mandibular advancement surgeries and purely while allowing the flexibility to avoid injuries at the system's mucosal emergence point. This flexibility is crucial to the comfort of both the patient and the orthodontist.

Indeed, all the reactivations of the system are done very quickly and are very simple to perform.

orthodontic corrections of dental class-III malocclusions will now be approached more calmly. We have decided to refer to this anchorage as an "Abalakov-type" anchorage to credit the person who inspired us to use it. 